\title{
Responses of Synechocystis sp. PCC 6803 to heterologous biosynthetic pathways
}

\author{
Konstantinos Vavitsas ${ }^{1}$, Emil Østergaard Rue ${ }^{1}$, Lára Kristín Stefánsdóttir² , Thiyagarajan Gnanasekaran ${ }^{1,3}$, \\ Andreas Blennow ${ }^{1}$, Christoph Crocoll ${ }^{1}$, Steinn Gudmundsson ${ }^{2}$ and Poul Erik Jensen ${ }^{1 *}$ (D)
}

\begin{abstract}
Background: There are an increasing number of studies regarding genetic manipulation of cyanobacteria to produce commercially interesting compounds. The majority of these works study the expression and optimization of a selected heterologous pathway, largely ignoring the wholeness and complexity of cellular metabolism. Regulation and response mechanisms are largely unknown, and even the metabolic pathways themselves are not fully elucidated. This poses a clear limitation in exploiting the rich biosynthetic potential of cyanobacteria.

Results: In this work, we focused on the production of two different compounds, the cyanogenic glucoside dhurrin and the diterpenoid 13R-manoyl oxide in Synechocystis PCC 6803. We used genome-scale metabolic modelling to study fluxes in individual reactions and pathways, and we determined the concentrations of key metabolites, such as amino acids, carotenoids, and chlorophylls. This allowed us to identify metabolic crosstalk between the native and the introduced metabolic pathways. Most results and simulations highlight the metabolic robustness of cyanobacteria, suggesting that the host organism tends to keep metabolic fluxes and metabolite concentrations steady, counteracting the effects of the heterologous pathway. However, the amino acid concentrations of the dhurrin-producing strain show an unexpected profile, where the perturbation levels were high in seemingly unrelated metabolites.
\end{abstract}

Conclusions: There is a wealth of information that can be derived by combining targeted metabolite identification and computer modelling as a frame of understanding. Here we present an example of how strain engineering approaches can be coupled to 'traditional' metabolic engineering with systems biology, resulting in novel and more efficient manipulation strategies.

Keywords: Cyanobacteria, Metabolism, Terpenoids, Amino acids, Metabolic modelling

\section{Background}

Metabolic engineering is the manipulation of living organisms to produce a desired product. This manipulation commonly refers to the alteration of the metabolism and the strain optimization required to increase production titers. Most of the metabolic engineering breakthroughs have taken place using heterotrophic model organisms, such as Escherichia coli and Saccharomyces cerevisiae; however, this limited choice of organisms restricts the application potentials, thus highlighting the

\footnotetext{
*Correspondence: peje@plen.ku.dk

${ }^{1}$ Copenhagen Plant Science Centre, Department of Plant

and Environmental Sciences, University of Copenhagen, Thorvaldsensvej

40, 1871 Frederiksberg C, Denmark

Full list of author information is available at the end of the article
}

need of expanding towards other organisms [1]. Cyanobacteria are promising biotechnological organisms that combine the capability to perform photosynthesis like higher plants, with simple, unicellular organization like E. coli and S. cerevisiae. The biosynthetic and metabolic properties of cyanobacteria, together with their facile genetic manipulation, allow for the production of various industrially interesting compounds [2-4].

Amino acids are among the building blocks of life, but they also have tremendous commercial importance, both as end-products and as precursor molecules for production of various complex biomolecules [5]. Aromatic amino acids, and their precursors in particular, are the starting points of many natural compounds, including flavonoids, glucosinolates, and lignins [2, 6]. Terpenoids 
are another diverse class of metabolic products. They consist of a carbon skeleton that is formed from one or multiple isoprenoid units, further decorated by modifying enzymes [7]. Their applications range from biofuels to anticancer and anti-malarial drugs. Isoprene has been produced in cyanobacteria in the $\mathrm{g} / \mathrm{L}$ of culture titer range $[8,9]$, and the biosynthetic pathway of the diterpenoid 13R-manoyl oxide-the precursor of the cAMP activator forskolin-was expressed in Synechocystis PCC 6803 (hereafter Synechocystis) [10]. More recently, we showed that Synechocystis can produce dhurrin, a cyanogenic glucoside, in a light-dependent manner, using tyrosine and UDP-glucose as precursor molecules [11].

At present, the product titers of most commercially interesting products obtained in cyanobacteria are low [2]. This may reflect a lack of knowledge on the effects of introducing foreign pathways in the host organisms. In this work, we focused on the production of dhurrin and on 13R-manoyl oxide in Synechocystis. The strategy was to employ genome-scale modelling to determine metabolic relationships and we experimentally determined the levels of key metabolites, with the aim to uncover responses that affect the optimal production of amino acid and isoprene derived compounds. Our data reveal specific metabolic crosstalk with the inserted pathways and provide important information on the mechanisms Synechocystis employs to regulate its metabolism.

\section{Methods}

\section{Strains, growth conditions and cell harvesting}

The Synechocystis strains used in this work are listed in Table 1. Cyanobacterial cultures were grown in $50 \mathrm{~mL}$ glass tubes (culture volume $20 \mathrm{~mL}$ ), in BG-11 media at $30{ }^{\circ} \mathrm{C}$, continuously supplied with $3 \% \mathrm{CO}_{2}$ enriched air and supplemented with $50 \mu \mathrm{g} / \mathrm{mL}$ spectinomycin. The light intensity was steady at $50 \mu \mathrm{mol}$ photons $/ \mathrm{s} / \mathrm{m}^{2}$. Cultures were grown for $24 \mathrm{~h}$ and then induced with $2 \mathrm{mM}$ isopropyl $\beta$-D-1-thiogalactopyranoside (IPTG). Cells were harvested after $48 \mathrm{~h}$ of growth by centrifugation at $14,000 \mathrm{~g}$ for $2 \mathrm{~min}$. The cell pellets were flash-frozen in liquid nitrogen and stored at $-80{ }^{\circ} \mathrm{C}$ until further use.

\section{Genetic modification of Synechocystis}

CfTPS2 [GenBank: KF444507] and CfTPS3 [GenBank: KF444508], encoding the diterpene synthases that convert geranylgeranyl diphosphate (GGPP) to $13 R$-manoyl oxide [13], were codon-optimized using the OptimumGene $^{\mathrm{TM}}$ optimization tool for Synechocystis, available from GenScript and inserted into the pDF-trc shuttle vector [12]. Vector construction and Synechocystis transformation were performed as described in [11]. Briefly, the two genes, each preceded by a ribosome binding site, were inserted as an operon into a linearized pDF-trc vector using T4 DNA ligase (New England BioLabs). Synechocystis was transformed via triparental mating [14]. The construction of the dhurrin strain was previously reported [11].

\section{Identification and quantification of $13 R$-manoyl oxide}

Cell pellets were homogenized in a Bullet Blender ${ }^{\circledR}$ homogenizer (Next Advance Inc.) in methanol using $0.15 \mathrm{~mm}$ zirconium oxide beads. This was followed by a hexane extraction for $3 \mathrm{~h}$ at room temperature at the Roto-Shake Genie ${ }^{\circledR}$ (Scientific Industries Inc.) revolving at $25 \mathrm{rpm}$. The presence of $13 R$-manoyl oxide in the extract was confirmed by gas chromatography-mass spectrometry (GC-MS) analysis using a GCMS-QP2010 Ultra (Shimadzu), and the method are described in [10]. Manoyl oxide was quantified using GC-FID in a SCION 436 GC-FID (Bruker) as described in [10], with the following modifications: $2 \mu \mathrm{L}$ sample was injected, and the amount of manoyl oxide was quantified via a standard curve.

\section{Pigment analysis}

The photosynthetic pigments were extracted from cell pellets in $90 \%(\mathrm{v} / \mathrm{v})$ methanol and analyzed by high performance liquid chromatography (HPLC) in a LC-20AT High Pressure Liquid Chromatographer with a SPDM20A Photodiode Array Detector (Shimadzu, Japan) as described in [15]. Pigments were identified by comparing retention times and absorption spectra with standard pigments (DHI, Hørsholm, Denmark), and their concentration determined via external authentic standards.

\section{LC-MS analysis for identification of dhurrin and amino acids}

Cell pellets were homogenized as described in "Identification and quantification of $13 R$-manoyl oxide" section in $80 \%(\mathrm{v} / \mathrm{v})$ methanol. Extracts were diluted to $20 \%(\mathrm{v} / \mathrm{v})$

Table 1 Synechocystis PCC 6803 strains used in this work

\begin{tabular}{ll}
\hline Strain & Information \\
\hline Control & Empty pDF-trc vector/spec ${ }^{R}$ \\
$\mathrm{DH}$ & Codon-optimized dhurrin biosynthetic pathway in an operon (CYP79A1, CYP71E1, and UGT85B1) in pDF-trc vector/spec ${ }^{R} \quad$ [11] \\
$\mathrm{MO}$ & Codon-optimized 13R-manoyl oxide biosynthetic pathway in an operon (CFTPS2 and CFTPS3) in pDF-trc vector/spec \\
\hline
\end{tabular}


methanol concentration and filtered using $0.22 \mu \mathrm{m}$ Ultrafree-MC GV Centrifugal Filter (Merck Millipore). The dhurrin analysis was performed as described in [11].

The amino acid analysis was performed as described in [16], adopted from a protocol by [17] originally described for aspartate-derived amino acids by [18]. Briefly, samples for amino acid quantification were spiked with ${ }^{13} \mathrm{C},{ }^{15} \mathrm{~N}$-labeled amino acids (Algal amino acids, Isotec, Miamisburg, USA) at a concentration of $1 \mu \mathrm{g} / \mathrm{mL}$ prior to analysis by LC-MS.

Chromatography was performed using an Advance UHPLC system (Bruker, Bremen, Germany). Separation was achieved using a Zorbax Eclipse XDB-C18 column $(50 \times 4.6 \mathrm{~mm}, 1.8 \mu \mathrm{m}$, Agilent Technologies, Germany) . Formic acid $(0.05 \%)$ in water and acetonitrile (supplied with $0.05 \%$ formic acid) were employed as mobile phases $A$ and $B$, respectively. The elution profile was: $0-1.5 \mathrm{~min}$, $3 \% \mathrm{~B}$ in $\mathrm{A} ; 1.5-3.1 \mathrm{~min}, 3-100 \% \mathrm{~B}$ in $\mathrm{A} ; 3.1-3.6 \mathrm{~min} 100 \%$ $\mathrm{B}, 3.6-3.9 \mathrm{~min} 100-3 \% \mathrm{~B}$ in $\mathrm{A}$ and $3.9-5.0 \mathrm{~min}$ in $3 \% \mathrm{~B}$. The mobile phase flow rate was $500 \mu \mathrm{L} / \mathrm{min}$. The column temperature was maintained at $40{ }^{\circ} \mathrm{C}$. The chromatography system was coupled to an EVOQ Elite TripleQuad mass spectrometer (Bruker, Bremen, Germany) equipped with an electrospray ion source operated in positive ionization mode. The instrument parameters were optimized by infusion experiments with pure standards (Amino acid standard mix, Fluka, St. Louis, USA). The ion spray voltage was maintained at $3000 \mathrm{~V}$. Cone temperature was set to $300{ }^{\circ} \mathrm{C}$ and cone gas to 20 psi. Heated probe temperature was set to $300{ }^{\circ} \mathrm{C}$ and probe gas flow set to 50 psi. Nebulizing gas was set to 60 psi and collision gas to 1.6 mTorr. Multiple reaction monitoring was used to monitor parent analyte ion $\rightarrow$ product ion transitions. Multiple reaction monitoring and corresponding collision energies can be found in [16]. Both Q1 and Q3 quadrupoles were maintained at unit resolution. Bruker MS Workstation software (Version 8.1.2, Bruker, Bremen, Germany) was used for data acquisition and processing.

\section{Glycogen quantification}

Glycogen concentration was determined by enzymatic hydrolysis of the cellular glycogen polysaccharide and quantifying the derived glucose. Pelleted cells were homogenized in a Bullet Blender ${ }^{\circledR}$ homogenizer (Next Advance Inc.). The broken cells were resuspended in a [10 $\mathrm{mM} \mathrm{K}_{2} \mathrm{PO}_{4}, 1 \mathrm{mM} \mathrm{CaCl}, 0.02 \% \mathrm{w} / \mathrm{v} \mathrm{NaN}$ ] buffer, preheated at $80{ }^{\circ} \mathrm{C}$. The samples were incubated for 20 min at $80{ }^{\circ} \mathrm{C}$ with the addition of $30 \mathrm{U}$ thermostable $\alpha$-amylase (Megazyme), and were subsequently incubated overnight with the addition of $7 \mathrm{U}$ pullulanase M1 and amyloglucosidase (Megazyme). The extracts were spun down, the supernatant filtered, and analyzed by High Pressure Anion-Exchange Chromatography using a Pulsed Amperometric Detection (HPAEC-PAD) BioLC system (Dionex, Sunnyvale, CA, USA) equipped with an AS50 autosampler, GS50 gradient pump and ED50 electrochemical detector. Samples $(10 \mu \mathrm{L})$ were injected and separation of glucose from other sample components was performed using a CarboPac ${ }^{\mathrm{TM}}$ SA10 column with a flow rate of $0.35 \mathrm{~mL} / \mathrm{min}$ and isocratic $1 \mathrm{mM} \mathrm{NaOH}$ as eluent. Peaks were evaluated using Chromeleon V.6.70 and glucose concentrations calculated by external authentic glucose standards.

\section{Oxygen evolution}

Cyanobacterial samples were collected $48 \mathrm{~h}$ after inoculation. Samples were diluted with fresh BG11 media to a concentration of $\sim 4 \mu \mathrm{g}$ chlorophyll $/ \mathrm{mL}$ and dark-adapted for $5 \mathrm{~min}$. Prior to measurements, $\mathrm{NaHCO}_{3}$ was added to the samples to a final concentration of $10 \mathrm{mM}$. Oxygen evolution was measured using a Clark-type electrode (LD2/3 Electrode chamber, Oxy-Lab Systems, Hansatech Instruments $\left.{ }^{\circledR}\right)$. Oxygen evolution was measured at increasing light intensities $(23,55,115,420,812$ and $1660 \mu \mathrm{mol} / \mathrm{m}^{2} / \mathrm{s}$ ) provided with a Schott lamp (HLX 64634 EFR 150 W Osram ${ }^{\circledR}$ Xenophot) with a 0.6 neutral density optic filter.

\section{Metabolic modelling}

The iJN678 metabolic network reconstruction of Synechocystis [19] version 1.1 was used to study the effects of expressing the heterologous pathways in silico. The model was updated to include the Entner-Doudoroff pathway [20], modifications in the tyrosine biosynthesis from arogenate according to [21] and corrections from [22]. The reactions involved in the heterologous pathways and the model updates are listed in Additional file 1: Table S1. As a result, three networks were obtained, one corresponding to the wild type which was used as a control, one corresponding to the dhurrin strain, and one to the manoyl oxide strain. The models were analyzed by randomly sampling feasible flux values from the networks $[23,24]$. Prior to random sampling, the metabolic networks were constrained with the experimental growth and product formation rates (lower bounds only, $2.3 \mu \mathrm{mol}$ dhurrin/gDW/h and $0.8 \mu \mathrm{mol}$ manoyl oxide/ gDW/h respectively). The complete set of model constraints is given in Additional file 1: Table S5 and the model is provided in SBML format in Additional file 3. Random sampling is an unbiased method in the sense that a biological objective (e.g. maximization of biomass) is not needed. The method results in a number of flux vectors representing feasible states of the network where the elements of a single flux vector correspond to fluxes in individual reactions. The mean and standard deviation of flux values were computed to enable comparison 
of fluxes in the heterologous pathways versus a control. For the models corresponding to the engineered strains, pairwise correlation coefficients were computed between all reactions in the network to enable identification of network reactions most strongly correlated with product formation [24]. The computational analysis was carried out in the Matlab environment (Mathworks) using the COBRA toolbox version 2.0 [25] and the Gurobi solver.

\section{Results}

\section{Expression of the two heterologous pathways}

Dhurrin is a cyanogenic glucoside derived from tyrosine, and its biosynthetic pathway involves two cytochrome $\mathrm{P} 450$ s that convert tyrosine into $p$-hydroxymandelonitrile and a UDP-glycosyltransferase that transfers a glucose from UDP-glucose (Fig. 1a). Dhurrin was produced in Synechocystis by inserting the three biosynthetic enzymes as an operon into the pDF-trc shuttle vector [11] (Fig. 1b). The second compound we chose is the forskolin precursor $13 R$-manoyl oxide, a diterpenoid derived from geranylgeranyl diphosphate by the enzymatic action of two diterpene synthases [13] (Fig. 1a). Synechocystis was previously engineered to produce manoyl oxide, where the diterpene synthases were incorporated in a genomic neutral site, under the transcriptional control of a $p s b A$ (aconstitutive promoter) and a nickel-inducible promoter [10]. In order to directly compare the effects of the two heterologous biosynthetic pathways, we expressed the manoyl oxide pathway in a way similar to the dhurrin one: we codon-optimized the two diterpene synthases and inserted them into the pDF-trc vector, thus expressing the two genes under the strong, IPTG-inducible trc promoter (Fig. 1b).

We used a combination of methods to study the response of Synechocystis to the two heterologous pathways. Both the dhurrin (DH) and the manoyl oxide (MO) producing strains exhibited slower growth rates (Fig. 2a). During the 1-day period, the control strain had an average doubling time of $11.5 \mathrm{~h}$, while the $\mathrm{DH}$ and $\mathrm{MO}$ strains had doubling times of 14.7 and $12.7 \mathrm{~h}$ respectively. The dhurrin strain displays a more acute growth reduction after the IPTG induction. This inverse relationship between growth and product formation for both the dhurrin and manoyl oxide strains is not surprising and has been observed previously, e.g. [9, 11, 26, 27] since the organism allocates fixed carbon and reducing power towards the new metabolite-resources that would otherwise be available for biomass accumulation.

As previously reported [11], the DH strain secretes dhurrin to the culture medium reaching a concentration of $4.2 \mathrm{mg} / \mathrm{L}$ after 2 days of growth (the product yield is the same as reported in [11] at the same time point). The newly-engineered $\mathrm{MO}$ strain produces the heterologous diterpenoid manoyl oxide, reaching titers of $2 \mathrm{mg} / \mathrm{L}$ ( $\sim 0.98 \mathrm{mg} / \mathrm{g}$ dry cell weight) of $13 R$-manoyl oxide after 2 days of growth (Fig. 2b). Normalized to cellular biomass, the manoyl oxide productivity increased more than twofold, compared to the previously reported $0.45 \mathrm{mg} / \mathrm{g}$ dry cell weight after 4 days of growth [10]. This is probably due to the different expression approach: in the current work a stronger promoter in a shuttle vector, as well as codon-optimized genes are used and in addition to the fact that the liquid cultures were supplemented with $3 \%$ $\mathrm{CO}_{2}$, which allows them to grow faster.

\section{Identification of correlated reactions}

The dhurrin and the manoyl oxide biosynthesis initiate from tyrosine and GGPP, respectively. The consumption of these metabolites can potentially lead to imbalances in the cellular metabolism, while affecting reactions and pathways that are not directly linked to the heterologous reactions. Manoyl oxide derives form geranylgeranyl diphosphate (GGPP), produced in cyanobacteria via the methylerythritol phosphate (MEP) pathway (Fig. 3). Interactions between reactions in the metabolic networks were analyzed in terms of correlation coefficients calculated from the randomly sampled flux values (Additional file 2: Table S6). The metabolic reactions that displayed the strongest correlation with manoyl oxide productivity are listed in Additional file 1: Table S2. They are mainly reactions involved in forming MEP and GGPP. The isopentenyl diphosphate (IPP) isomerase reaction is computationally predicted to be negatively correlated with manoyl oxide secretion, highlighting the need of a balanced production of isopentenyl diphosphate (IPP) and dimethylallyl diphosphate (DMAPP). IPP and DMAPP react to form a geranyl diphosphate (GPP) molecule, which can be converted to GGPP with the addition of two IPP molecules (via farnesyl diphosphate) (Fig. 3). As a consequence, a proper equilibrium between IPP and DMAPP levels (namely 3:1) is required to maximize the GGPP formation. The enzyme that balances the two metabolite levels is the IPP isomerase, which reversibly converts IPP to DMAPP.

Dhurrin derives from tyrosine, which in turn originates from the shikimate pathway (Fig. 3). Correlation coefficients derived from random sampling show the dependence of dhurrin accumulation on flux in the shikimate pathway, as well as a connection with sugar catabolism and anabolism (glucose-1-phosphate uridylyltransferase activity correlates positively and phosphoglucomutase activity correlates negatively with dhurrin formation) (Additional file 1: Table S3).

Comparison of the predicted flux values in the modified strains with the predicted fluxes in the control strain (Fig. 3) shows that, in order to achieve the reported 
a<smiles>NC(Cc1ccc(O)cc1)C(=O)O</smiles>

$p$-hydroxyphenylacetaldoxime CYP71E1<smiles>N#CC(O)c1ccc(O)cc1</smiles>
p- hydroxymandelonitrile

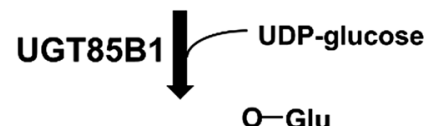<smiles>COC(C#N)c1ccc(O)cc1</smiles>

Dhurrin

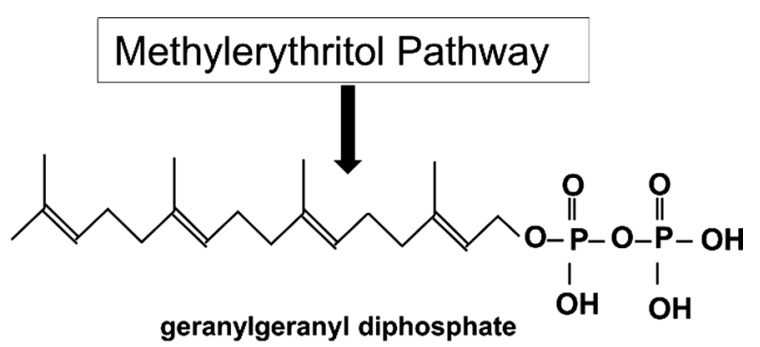

geranylgeranyl diphosphate

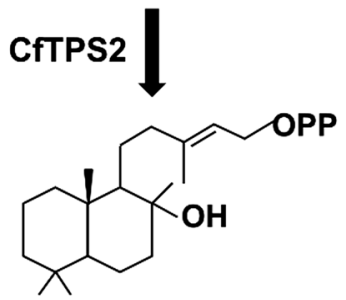

Copal-8-ol-diphosphate

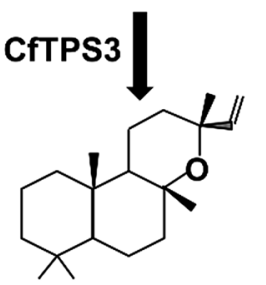

(13R) - Manoyl oxide

b
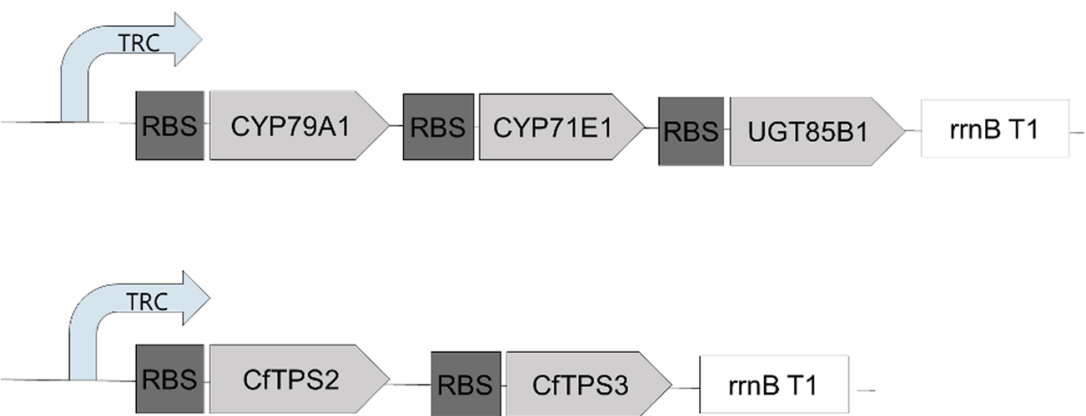

Fig. 1 Dhurrin and 13R-manoyl oxide biosynthetic pathways and the genetic constructs for their expression in Synechocystis. a The precursors tyrosine and geranylgeranyl diphosphate are derived from the shikimate and the methylerythritol pathway respectively. The final glycosylation step in dhurrin biosynthesis connects the cyanogenic glucoside formation with the sugar metabolism. $\mathbf{b}$ The genes encoding enzymes of the dhurrin and the manoyl oxide biosynthetic pathways, respectively, were arranged in operons, under the control of the trc promoter (TRC). The ribosome binding site (RBS) ribo* [39] is incorporated before the $5^{\prime}$ of each gene, and each operon concludes with the rrnB terminator (rrnB T1). Both linear constructs were inserted in the pDF-trc expression vector

productivity for manoyl oxide, the flux from the MEP pathway requires an approximately threefold increase. The shikimate pathway also needs to increase its output by approximately $20 \%$. It is also noteworthy that the increase in both the precursor pathways are predicted to be such as to roughly cover the extra demand posed 

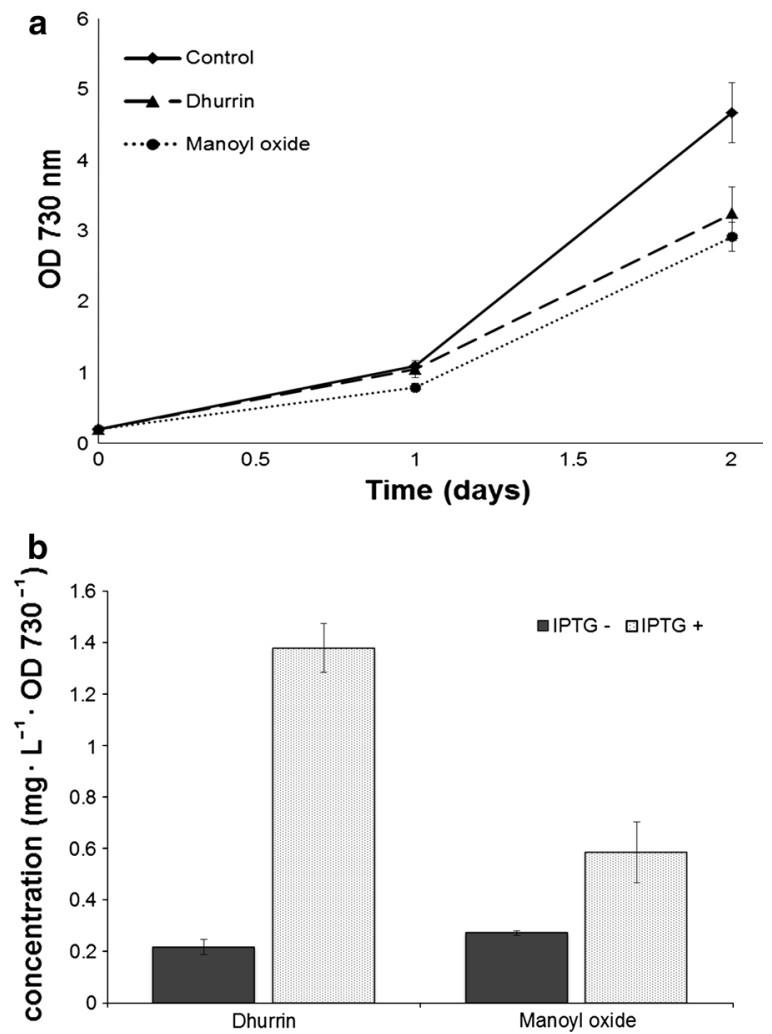

Fig. 2 Growth rates and production titers of the two cyanobacterial strains, producing dhurrin and $13 R$-manoyl oxide. The error bars represent the standard deviation within three biological replicates. a Initial growth rate is represented as a function of the optical density at $730 \mathrm{~nm}$. Cells were grown in air supplemented with $3 \% \mathrm{CO}_{2}$, and were induced with 2 mM IPTG $24 \mathrm{~h}$ after inoculation. b Dhurrin (DH) and $13 R$-manoyl oxide $(\mathrm{MO})$ accumulation at $48 \mathrm{~h}$ of growth, with and without IPTG induction

by the heterologous reaction, while not affecting severely the fluxes of the branching pathways (Fig. 3).

\section{Effects on amino acids, carotenoids, and oxygen evolution}

The role of aromatic amino acids in dhurrin formation and the computational predictions described above prompted us to determine the amino acid concentration of the both the manoyl oxide and dhurrin strains and compare these to the empty vector control (Additional file 1: Table S4). Among the 17 amino acids analyzed in the dhurrin strain we observed that the phenylalanine, tryptophan, and tyrosine concentrations were similar to the control (Fig. 4). However, most of the other amino acids accumulated in a much higher concentrations. In contrast, the manoyl oxide-producing strain did not display any dramatic difference in the amino acid content when compared to the empty vector control. The minor decrease in phenylalanine and tryptophan could be due to the overall decrease in growth (Fig. 2a).
These data suggest the presence of a response or regulatory mechanism that causes this non-predicted amino acid variation in the dhurrin strain. One could consider a scenario where there is an increase in the breakdown of storage carbohydrates. In the experiments, the strains were grown in high $\mathrm{CO}_{2}$ concentration, therefore they should have accumulated a large amount of glycogen [28]. After IPTG induction, the increased consumption of tyrosine for dhurrin biosynthesis will lead to increased erythrose-4P and phosphoenolpyruvate consumption, both of which participate in the central carbon metabolism (Fig. 4 insert). If the response to this perturbation is an increase in glycolysis, this could replenish the carbon pool. Consequently, the amino acid precursors would accumulate, leading to the increase in amino acids that was observed. Such effects have been reported previously, where the knockout of the glycogen synthase in Synechococcus elongatus PCC 7002 led to an over-accumulation of the TCA cycle metabolites [29], while an increased glycogen breakdown led to increased amino acid quantities [30]. We tested this hypothesis by quantifying the glycogen content in both the $\mathrm{DH}$ and the $\mathrm{MO}$ production strains and compared it to the empty vector control. Our results did not demonstrate any significant difference between the three strains in the glycogen content (Additional file 1: Figure S1), making a mechanism based on storage carbohydrate breakdown less likely. A potential reason for this could be that the sudden decrease in growth rate following IPTG inductionwhich is more pronounced than what we would expect solely by the redirection of metabolic resources towards dhurrin-causes an imbalance in carbon homeostasis, thereby perturbing the metabolic profile of the organism.

Cyanobacteria rely on photosynthesis as an energy generator, and a variation in photosynthetic activity would affect many cellular and metabolic functions. Moreover, a significant upregulation of photosynthesis could explain a generic increase in the TCA cycle intermediates, and consequently an increase in amino acid levels. The amount of oxygen evolved by the cells provides an indication of differences in this aspect. Oxygen evolution was not found to be significantly affected when comparing the dhurrin-producer with the control (Fig. 5a). The manoyl oxide strain evolved slightly less oxygen; however, the differences were not significant and therefore the architecture and functionality of the photosynthetic apparatus is likely unaffected. The same small decrease in $\mathrm{O}_{2}$ evolution was observed in Synechococcus PCC 7492 during the production of the monoterpene limonene [31].

Carotenoids share the same precursor-GGPP-with manoyl oxide and we investigated whether the new competing pathway affect the pigmentation. We quantified 


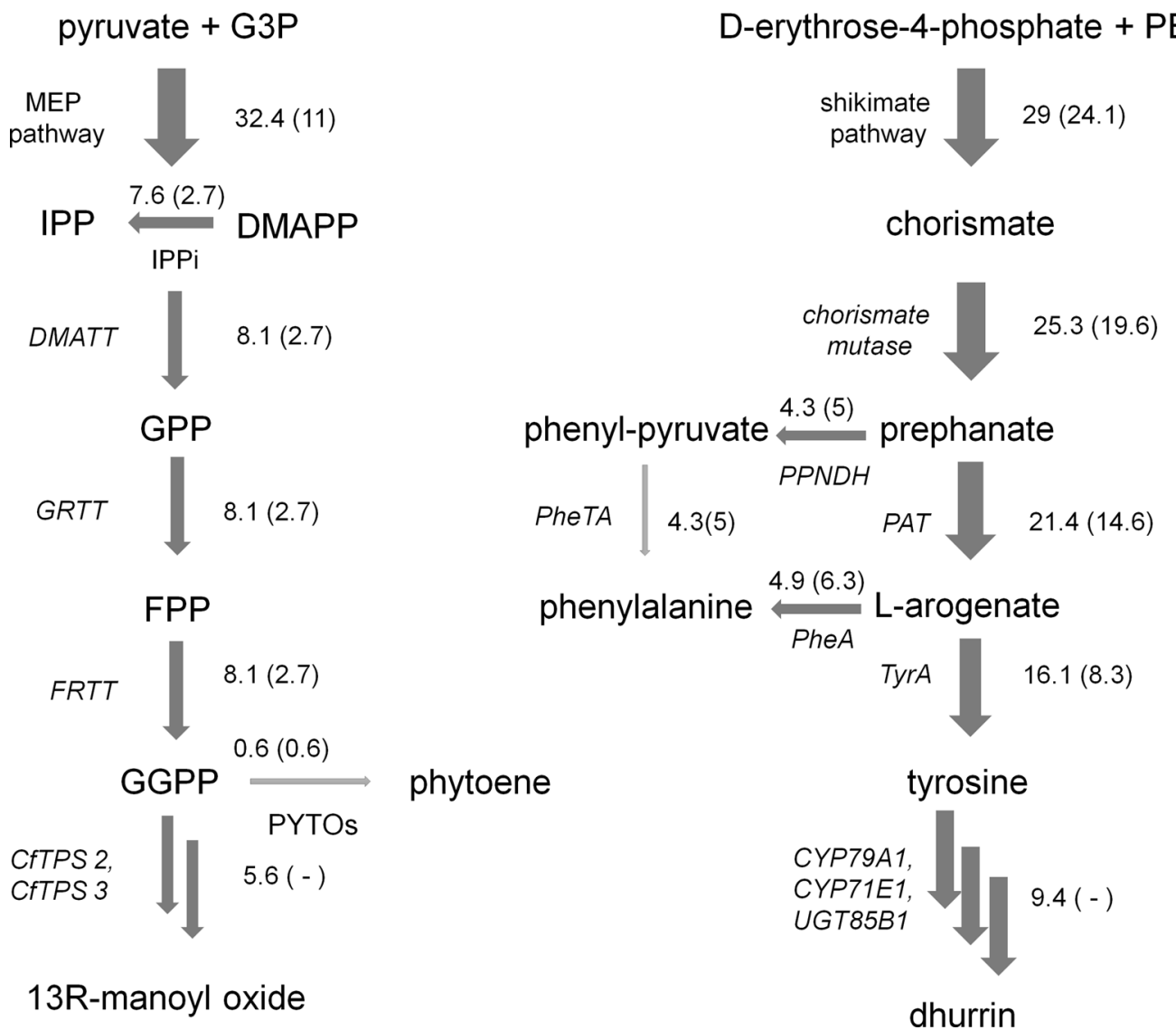

Fig. 3 Predicted average fluxes of the biosynthetic pathways towards manoyl oxide (left) and dhurrin (right). The values were determined by random sampling ( $\mu \mathrm{mol}$ product/gDW/h) in the presence and the absence of the heterologous biosynthetic pathway (values outside and inside the brackets, respectively). G3P glyceraldehyde 3-phosphate, IPP isopentenyl diphosphate, DMAPP dimethylallyl diphosphate, GPP geranyl diphosphate, FPP farnesyl diphosphate, GGPP geranylgeranyl diphosphate, PEP phosphoenolpyruvate

the major photosynthetic pigments using extraction followed by HPLC analysis, and we see that indeed the cells were not affected in this aspect (Fig. 5b). The flux towards carotenoids was generally small, as indicated by the in silico flux analysis, and it was predicted to remain steady in the presence of the manoyl oxide biosynthetic pathway (Fig. 3).

\section{Discussion}

The amount of fixed electrons directed towards dhurrin and manoyl oxide was 0.24 and $0.28 \%$ of the total fixed electrons respectively, determined by the ratio of the product titers and the biomass, as described in [2]. These numbers are small and appear to be well within the buffering capacity of the photosynthetic regulatory mechanisms. In support of this view, previous work [11, 32, 33], together with results presented here, indicate that the expression of cytochrome P450s does not drain a significant number of electrons out of the photosynthetic electron transfer chain. Dhurrin and manoyl oxide, being the product of long and complex metabolic routes, require a large amount of fixed carbons and subsequently harvested photons, as compared to simpler products, such as ethanol and succinic acid. Fixed carbon and its proper channeling appear to be the main restrictions for increased productivity, and not electron redirection, due to the versatility of the redox power distribution proteins [34].

Synechocystis displays a remarkable robustness, and is very recalcitrant to extended metabolic manipulations. Despite the fact that it is relatively straightforward to express a heterologous pathway and fine-tune expression levels and fluxes within the pathway itself, there are very few examples in the literature where high production titers have been achieved. Moreover, there is a lack of specific knowledge about how cyanobacteria regulate their metabolism. Cyanobacteria tend to compensate for large perturbations, and pinpointing the way they do that is crucial for successful genetic engineering. System biology approaches, namely-omics work coupled with computational methodology, can facilitate 


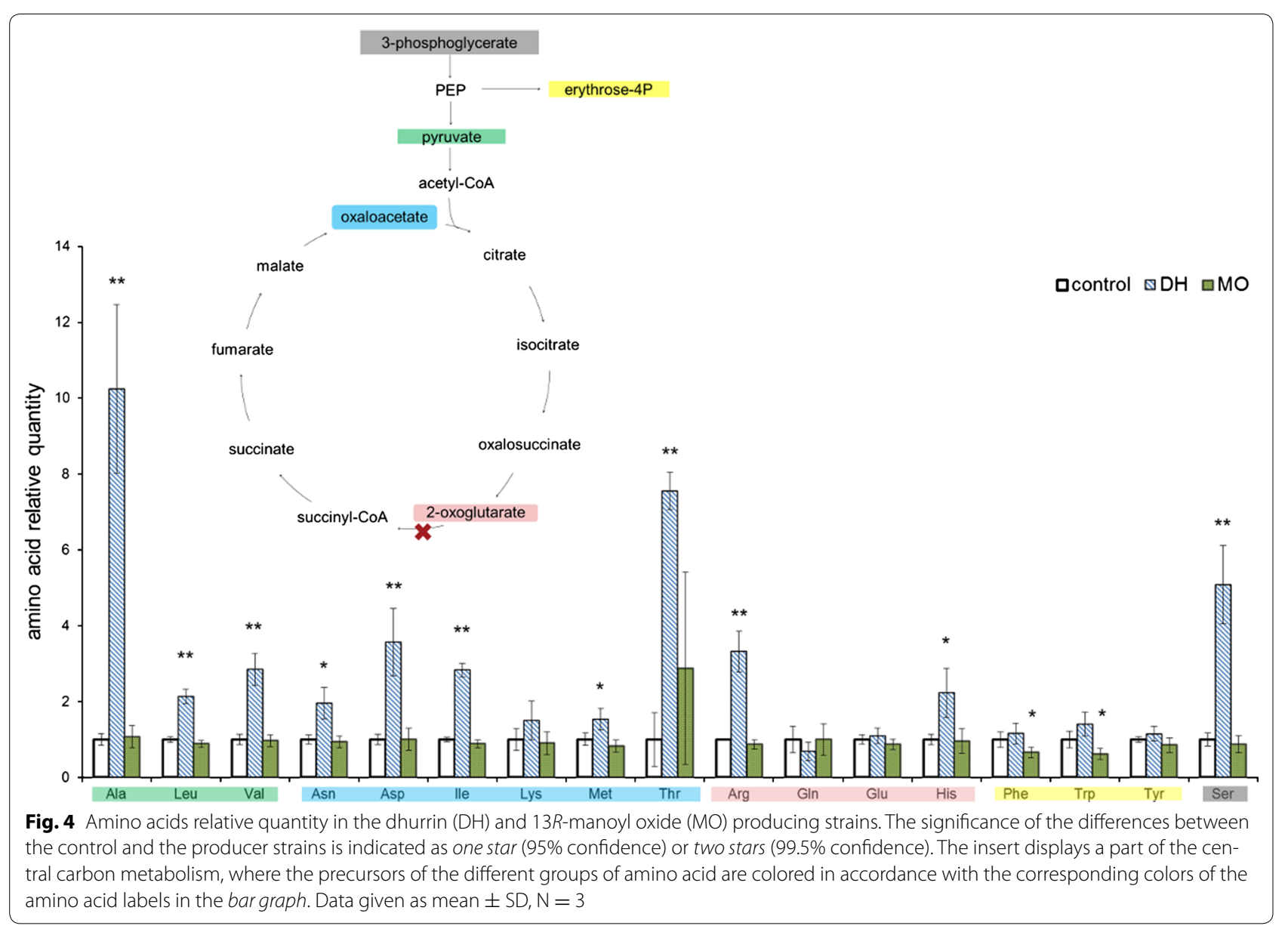

such an effort by locating connected metabolic pathways, identifying response mechanisms and off-target effects, and designing further improvement strategies [35]. Metabolic modelling and targeted metabolomics can quickly give valuable information on plausible effects of a given genetic modification, before more elaborate and resource-consuming methodologies, such as RNA sequencing and global metabolomics, are applied.

In this work we combined experimental and computational methods to understand how Synechocystis responds to the production of a diterpenoid and a cyanogenic glucoside. For both metabolites, the importance of channeling resources towards the biosynthesis of the precursor molecules is apparent, especially within the robust cyanobacterial metabolism. For dhurrin, there is a correlation with the catabolic reactions, while for the tyrosine derivatives resveratrol, $p$-coumaric acid, and caffeic acid, deletion of competing pathways, scaffolding, and expression optimization have previously been demonstrated efficient to increase production [36, 37]. Manoyl oxide production relies more on the balance between the different terpenoid metabolism branches and the efficient channeling through the MEP pathway. The overexpression of key enzymes of the GGPP biosynthesis has previously led to an increase of diterpenoid production in Nicotiana benthamiana leaves [15, 38], however this strategy was not successful when applied in Synechocystis [10]. Nevertheless, the careful study of the MEP pathway, the identification of the metabolic bottlenecks, and the optimization of fluxes via the screening of enzyme variants and enzyme fusions led to the high production of isoprene and limonene titers in Synechococcus PCC $7492[9,31]$. Such a strategy can be generally applied to increase terpenoid production in cyanobacteria.

\section{Conclusions}

We here present an example of how strain engineering approaches can be coupled to 'traditional' metabolic engineering with systems biology, resulting in better understanding of the organism as a system and potentially in novel and more efficient manipulation strategies. The insights acquired are applicable to a large number of other compounds that share biosynthetic routes and precursor molecules. We propose that the combination of metabolic 

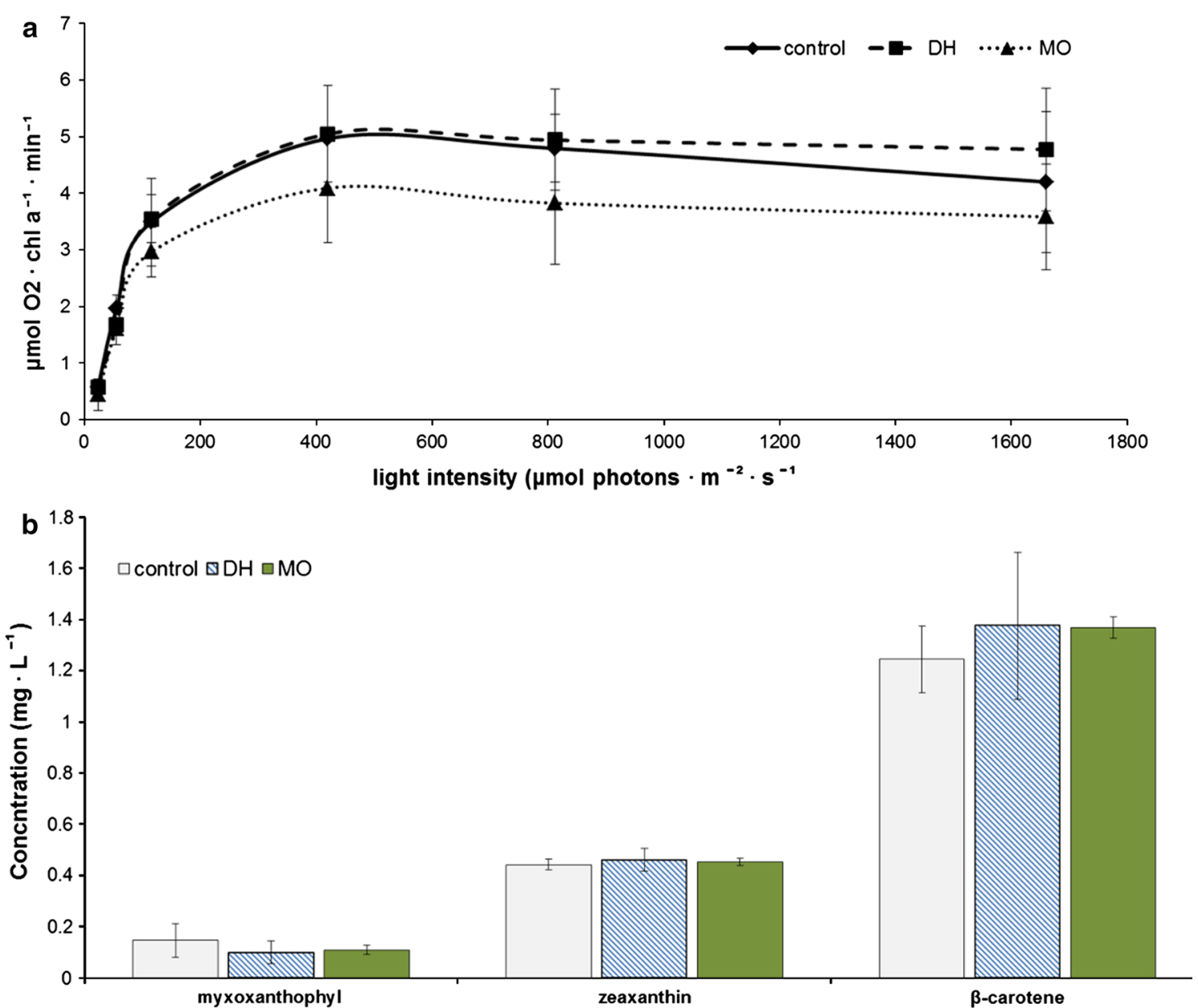

Fig. 5 Oxygen evolution and carotenoid content in the dhurrin $(\mathrm{DH})$ and $13 R$-manoyl oxide $(\mathrm{MO})$ producing strains. a Oxygen evolution of the three strains, normalized to chlorophyll a content. b The three most abundant carotenoids were quantified with HPLC. Data given as mean \pm SD, $N=3$

modelling and metabolite analysis is an approach that can be adjusted to the production of any heterologous compound, forming a frame for understanding of the cyanobacterial cell as a microbial cell factory.

\section{Additional files}

Additional file 1: Table S1. Reactions added to version 1.1 of the iJN678 model. Table S2. Reactions most strongly correlated with manoyl oxide production as determined by random sampling. Table S3. Reactions most strongly correlated with dhurrin production as determined by random sampling. Table S4. Amino acid concentrations ( $\mu \mathrm{mol} / \mathrm{mL} / \mathrm{OD} 730$ ) of the control, dhurrin, and 13-R manoyl oxide producing strains. Table S5. Constraints applied in the three metabolic models used in this work. Figure S1. Glycogen content in the dhurrin $(\mathrm{DH})$ and $13 R$-manoyl oxide (MO) producing strains compared to control (empty).

Additional file 2: Table S6. Average fluxes of reactions models, determined with random sampling.

Additional file 3. Updated metabolic models.

\section{Abbreviations}

G3P: glyceraldehyde 3-phosphate; IPP: isopentenyl diphosphate; DMAPP: dimethylallyl diphosphate; GPP: geranyl diphosphate; FPP: farnesyl diphosphate; GGPP: geranylgeranyl diphosphate; PEP: phosphoenolpyruvate; DH: dhurrin; MO: 13R-manoyl oxide; MEP: methylerythritol phosphate pathway; IPTG: isopropyl $\beta$-D-1-thiogalactopyranoside; GC-MS: chromatography-mass spectrometry; HPLC: high performance liquid chromatography.

\section{Authors' contributions}

$\mathrm{KV}$ planned and performed the experiments and the calculations, analyzed the data, and wrote the manuscript; $E \varnothing R, T G, A B, C C$ performed the experiments and reviewed the manuscript; LKS performed the calculations and reviewed the manuscript; SG and PEJ planned and performed the experiments and the calculations and reviewed the manuscript. All authors read and approved the final manuscript.

\section{Author details}

${ }^{1}$ Copenhagen Plant Science Centre, Department of Plant and Environmental Sciences, University of Copenhagen, Thorvaldsensvej 40, 1871 Frederiksberg C, Denmark. ${ }^{2}$ Center for Systems Biology, University of Iceland, Sturlugata 8 , 101 Reykjavik, Iceland. ${ }^{3}$ Present Address: ISBP-INSA de Toulouse, Avenue de Rangueil, 31077 Toulouse, France. 


\section{Acknowledgements}

The authors would like to thank Dr. Federico Cozzi for helping with the analytical equipment and Dr. Lars Scharff for fruitful discussions.

\section{Competing interests}

The authors declare that they have no competing interests.

\section{Availability of data and materials}

All data generated or analyzed during this study are included in this published article and its additional files.

\section{Consent for publication}

Not applicable.

\section{Ethics approval and consent to participate}

Not applicable.

\section{Funding}

The work was financially supported by: (1) Copenhagen Plant Science Centre, (2) "Plant Power: Light-Driven Synthesis of Complex Terpenoids Using Cytochromes P450" (12-131834) funded by Innovation Fund Denmark (previously the Danish Council for Strategic Research), (3) from the Novo Nordisk Foundation (Sustainable production of forskolin, a high-value diterpenoid, NNF13OC0005685), (4) the VILLUM Foundation (Light-driven biosynthesis: Improving photosynthesis by designing and exploring novel electron transfer pathways, Project No. 13363), (5) the COST Action ES1408 European network for algal-bioproducts (EUALGAE), (6) Danish National Research Foundation (DNRF) grant99; and (7) the University of Iceland Research Fund.

\section{Publisher's Note}

Springer Nature remains neutral with regard to jurisdictional claims in published maps and institutional affiliations.

Received: 22 May 2017 Accepted: 9 August 2017

Published online: 15 August 2017

\section{References}

1. Adams BL. The next generation of synthetic biology chassis: moving synthetic biology from the laboratory to the field. ACS Synth Biol. 2016:5:1328-30.

2. Nielsen AZ, Mellor SB, Vavitsas K, Wlodarczyk AJ, Gnanasekaran T, de Perestrello Ramos $\mathrm{H}$, Jesus $\mathrm{M}$, et al. Extending the biosynthetic repertoires of cyanobacteria and chloroplasts. Plant J. 2016;87:87-102.

3. Lau N, Matsui M, Abdullah AA. Cyanobacteria: photoautotrophic microbial factories for the sustainable synthesis of industrial products. Biomed Res Int. 2015:1-9.

4. Gangl D, Zedler JAZ, Rajakumar PD, Martinez EMR, Riseley A, Włodarczyk A, et al. Biotechnological exploitation of microalgae. J Exp Bot. 2015:66:6975-90

5. Becker J, Wittmann C. Systems and synthetic metabolic engineering for amino acid production - the heartbeat of industrial strain development. Curr Opin Biotechnol. 2012;23:718-26

6. Lee J-H, Wendisch VF. Biotechnological production of aromatic compounds of the extended shikimate pathway from renewable biomass. J Biotechnol. 2016. doi:10.1016/j.jbiotec.2016.11.016.

7. Davies FK, Jinkerson RE, Posewitz MC. Toward a photosynthetic microbial platform for terpenoid engineering. Photosynth Res. 2015;123:265-84.

8. Lindberg P, Park S, Melis A. Engineering a platform for photosynthetic isoprene production in cyanobacteria, using Synechocystis as the model organism. Metab Eng. 2010;12:70-9.

9. Gao X, Gao F, Liu D, Zhang H, Nie X, Yang C. Engineering the methylerythritol phosphate pathway in cyanobacteria for photosynthetic isoprene production from $\mathrm{CO}_{2}$. Energy Environ Sci. 2016;9:1400-11.

10. Englund E, Andersen-Ranberg J, Miao R, Hamberger B, Lindberg P. Metabolic engineering of Synechocystis sp. PCC 6803 for production of the plant diterpenoid manoyl oxide. ACS Synth Biol. 2015:4:1270-8.
11. Wlodarczyk A, Gnanasekaran T, Nielsen AZ, Zulu NN, Mellor SB, Luckner $M$, et al. Metabolic engineering of light-driven cytochrome P450 dependent pathways into Synechocystis sp. PCC 6803. Metab Eng. 2016;33:1-11.

12. Guerrero F, Carbonell VV, Cossu M, Correddu D, Jones PR. Ethylene synthesis and regulated expression of recombinant protein in Synechocystis sp. PCC 6803. PLoS ONE. 2012;7:e50470.

13. Pateraki I, Andersen-Ranberg J, Hamberger B, Heskes AM, Martens HJ, Zerbe $\mathrm{P}$, et al. Manoyl oxide (13R), the biosynthetic precursor of forskolin, is synthesized in specialized root cork cells in Coleus forskohlii. Plant Physiol. 2014;164:1222-36.

14. Huang $\mathrm{H}-\mathrm{H}$, Camsund D, Lindblad $\mathrm{P}$, Heidorn T. Design and characterization of molecular tools for a Synthetic Biology approach towards developing cyanobacterial biotechnology. Nucleic Acids Res. 2010;38:2577-93.

15. Gnanasekaran T, Vavitsas $K$, Andersen-Ranberg J, Nielsen AZ, Olsen CE, Hamberger B, et al. Heterologous expression of the isopimaric acid pathway in Nicotiana benthamiana and the effect of $\mathrm{N}$-terminal modifications of the involved cytochrome P450 enzyme. J Biol Eng. 2015;9:24.

16. Mirza N, Crocoll C, Erik Olsen C, Ann Halkier B. Engineering of methionine chain elongation part of glucoraphanin pathway in E. coli. Metab Eng 2016:35:31-7.

17. Docimo T, Reichelt M, Schneider B, Kai M, Kunert G, Gershenzon J, et al. The first step in the biosynthesis of cocaine in Erythroxylum coca: the characterization of arginine and ornithine decarboxylases. Plant Mol Biol. 2012;78:599-615.

18. Jander G, Norris SR, Joshi V, Fraga M, Rugg A, Yu S, et al. Application of a high-throughput HPLC-MS/MS assay to Arabidopsis mutant screening evidence that threonine aldolase plays a role in seed nutritional quality. Plant J. 2004;39:465-75

19. Nogales J, Gudmundsson S, Knight EM, Palsson BO, Thiele I. Detailing the optimality of photosynthesis in cyanobacteria through systems biology analysis. Proc Natl Acad Sci. 2012;109:2678-83.

20. Chen X, Schreiber K, Appel J, Makowka A, Fähnrich B, Roettger M, et al. The Entner-Doudoroff pathway is an overlooked glycolytic route in cyanobacteria and plants. Proc Natl Acad Sci. 2016;113:5441-6.

21. Bonner CA, Jensen RA, Gander JE, Keyhani NO. A core catalytic domain of the TyrA protein family: arogenate dehydrogenase from Synechocystis. Biochem J. 2004;382:279-91.

22. Shabestary K, Hudson EP. Computational metabolic engineering strategies for growth-coupled biofuel production by Synechocystis. Metab Eng Commun. 2016:3:216-26.

23. Palsson BO. Systems biology: constraint-based reconstruction and analysis. Cambridge: Cambridge University Press; 2015.

24. Schellenberger J, Palsson BO. Use of randomized sampling for analysis of metabolic networks. J Biol Chem. 2009:284:5457-61.

25. Schellenberger J, Que R, Fleming RMT, Thiele I, Orth JD, Feist AM, et al. Quantitative prediction of cellular metabolism with constraint-based models: the COBRA Toolbox v2.0. Nat Protoc. 2011;6:1290-307.

26. Oliver JWK, Machado IMP, Yoneda H, Atsumi S. Cyanobacterial conversion of carbon dioxide to 2,3-butanediol. Proc Natl Acad Sci. 2013;110:1249-54.

27. Kanno M, Carroll AL, Atsumi S. Global metabolic rewiring for improved $\mathrm{CO}_{2}$ fixation and chemical production in cyanobacteria. Nat Commun. 2017:8:14724

28. Schwarz D, Orf I, Kopka J, Hagemann M. Effects of inorganic carbon limitation on the metabolome of the Synechocystis sp. PCC 6803 mutant defective in glnB encoding the central regulator PII of cyanobacterial C/N acclimation. Metabolites. 2014:4:232-47.

29. Davies FK, Work VH, Beliaev AS, Posewitz MC. Engineering limonene and bisabolene production in wild type and a glycogen-deficient mutant of Synechococcus sp. PCC 7002. Front Bioeng Biotechnol. 2014;2:21.

30. Osanai T, Oikawa A, Numata K, Kuwahara A, lijima H, Doi Y, et al. Pathwaylevel acceleration of glycogen catabolism by a response regulator in the cyanobacterium Synechocystis species PCC 6803. Plant Physiol. 2014;164:1831-41.

31. Wang X, Liu W, Xin C, Zheng Y, Cheng Y, Sun S, et al. Enhanced limonene production in cyanobacteria reveals photosynthesis limitations. Proc Nat Acad Sci. 2016;113:14225-30.

32. Berepiki A, Hitchcock A, Moore CM, Bibby TS. Tapping the unused potential of photosynthesis with a heterologous electron sink. ACS Synth Biol. 2016;5:1369-75. 
33. Lassen LM, Nielsen AZ, Olsen CE, Bialek W, Jensen $K$, Møller BL, et al. Anchoring a plant cytochrome P450 via PsaM to the thylakoids in Synechococcus sp. PCC 7002: evidence for light-driven biosynthesis. PLoS ONE. 2014;9:e102184.

34. Mellor SB, Vavitsas K, Nielsen AZ, Jensen PE. Photosynthetic fuel for heterologous enzymes: the role of electron carrier proteins. Photosynth Res. 2017. doi:10.1007/s11120-017-0364-0.

35. Gudmundsson S, Nogales J. Cyanobacteria as photosynthetic biocatalysts: a systems biology perspective. Mol BioSyst. 2015;11:60-70.

36. Ni J, Tao F, Wang Y, Yao F, Xu P. A photoautotrophic platform for the sustainable production of valuable plant natural products from $\mathrm{CO}_{2}$. Green Chem. 2016;18:3537-48.
37. Xue Y, Zhang Y, Cheng D, Daddy S, He Q. Genetically engineering Synechocystis sp. pasteur culture collection 6803 for the sustainable production of the plant secondary metabolite $p$-coumaric acid. Proc Natl Acad Sci. 2014;111:1-6.

38. Brückner K, Tissier A. High-level diterpene production by transient expression in Nicotiana benthamiana. Plant Methods. 2013;9:46.

39. Heidorn T, Camsund D, Huang H-H, Lindberg P, Oliveira P, Stensjö K, et al. Synthetic biology in cyanobacteria engineering and analyzing novel functions. Methods Enzymol. 2011;497:539-79.

\section{Submit your next manuscript to BioMed Central and we will help you at every step:}

- We accept pre-submission inquiries

- Our selector tool helps you to find the most relevant journal

- We provide round the clock customer support

- Convenient online submission

- Thorough peer review

- Inclusion in PubMed and all major indexing services

- Maximum visibility for your research

Submit your manuscript at www.biomedcentral com/submit 